\title{
Blood lipid profiles following nonfocused ultrasonic treatment for noninvasive body contouring
}

\author{
Takashi Honda', Katsuhiro Kure ${ }^{1}$, Hiroyuki Goto', Takuya Suzuki², Mariko Mogami \\ Tsukasa Isago ${ }^{3}$ \\ ${ }^{1}$ Department of Plastic and Aesthetic Surgery, Tokyo Women's Medical University, Medical Center East, Tokyo 116-0013, Japan. \\ ${ }^{2}$ Clinical laboratory, Tokyo Women's Medical University, Medical Center East, Tokyo 116-0013, Japan. \\ ${ }^{3}$ Department of Plastic and Reconstructive Surgery, Tokyo Women's Medical University, Medical Center East, Tokyo 116-0013, Japan.
}

Address for correspondence: Dr. Takashi Honda, Department of Plastic and Aesthetic Surgery, Tokyo Women's Medical University, Medical Center East, 2-20-1 Nishinippori Arakawa-ku, Tokyo 116-0013, Japan. E-mail: honda.takashi@twmu.ac.jp

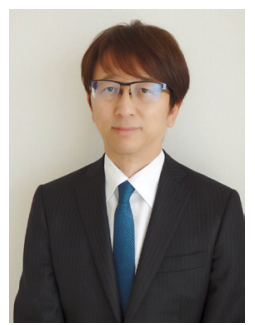

Dr. Takashi Honda is Associate Professor in the Department of Plastic and Aesthetic Surgery at Tokyo Womens' Medical University, Medical Center East. His field of expertise includes facial cosmetic surgery, maxillofacial reconstruction, aesthetic laser therapy, and breast surgery including augmentation, reduction and reconstruction.

\section{ABSTRACT}

Aim: Blood lipid profiles changed following nonfocused ultrasound treatments for body contouring. The present study elucidates clinical effects of these devices on adipose tissue. Methods: Ultrasound treatments for 5 males and 5 females in a supine position, ages 37-67 years, were applied at $20 \mathrm{KHz}$ and $3.0 \mathrm{~W} / \mathrm{cm}^{2}$ in modulated emission. Whole abdomen was treated by an ultrasound handpiece for $30 \mathrm{~min}$ followed by a 6-min lymphatic drainage. Waist circumferences at the level of the umbilicus and body weight were measured before and immediately after treatment. Blood lipid profiles including total cholesterol, low-density lipoprotein, high-density lipoprotein, triglyceride (TG), nonesterified fatty acid (NEFA) and lipoprotein-a were measured at baseline, every 10 min during treatment, and 15, 30, 45, 60, 75, and 90 min after treatment completion. Results: NEFA showed statistically higher values after $10 \mathrm{~min}$ following treatment initiation. Subsequent values remained high despite some fluctuation, reaching a maximum at $90 \mathrm{~min}$. In contrast, TG gradually decreased in concentration until the last measurement, especially for the first $30 \mathrm{~min}$, with statistically significant reduction. Changes in other lipid profiles and lipoprotein-a were not significant. Conclusion: Changes in NEFA concentration were significant following ultrasound treatment, and suggest that metabolism of TGs stored within the adipocytes occurred immediately after treatment initiation.

Key words:

Noninvasive body contouring; MC1 device; nonfocused ultrasound; cavitation; blood lipid profiles

\begin{tabular}{|l|l|}
\hline \multicolumn{2}{|c|}{ Access this article online } \\
\hline Quick Response Code: & Website: \\
\hline & http://parjournal.net \\
\cline { 1 - 2 } & \\
\cline { 2 - 3 } & DOI: \\
\hline
\end{tabular}

This is an open access article distributed under the terms of the Creative Commons Attribution-NonCommercial-ShareAlike 3.0 License, which allows others to remix, tweak and build upon the work non-commercially, as long as the author is credited and the new creations are licensed under the identical terms.

For reprints contact: service@oaepublish.com

How to cite this article: Honda T, Kure K, Goto H, Suzuki T, Mogami M, Isago T. Blood lipid profiles following nonfocused ultrasonic treatment for noninvasive body contouring. Plast Aesthet Res 2016;3:107-10

Received: 28-05-2015; Accepted: 30-12-2015 


\section{INTRODUCTION}

Nonsurgical body contouring devices, including cryolipolysis, low-level laser therapy, radiofrequency instruments, and external ultrasound devices, are widely used noninvasive procedures for body contouring and fat reduction. In a clinical environment, the most effective treatments for noninvasive fat reduction involve ultrasound, using either focused or nonfocused waves depending on how ultrasonic energy is delivered to the tissue. ${ }^{[1-4]}$

The MC1 instrument (General Product S.r.I., Montespertoli, Italy) uses nonfocused ultrasound, and is designed to induce stable cavitation while reducing adipose tissue volume in treated tissue. ${ }^{[2,5,6]}$

Despite its apparent clinical efficacy, biological mechanisms reducing adipose tissue are not fully understood. Adipose cell cavitation induced focal alterations of the plasma membrane and lipid leakage during in vivo porcine studies. ${ }^{|5|}$ Bani et al..$^{[6]}$ reported that ultrasound cavitation induced a statistically significant reduction in the size of adipocytes, the appearance of micropores, and triglyceride (TG) leakage.

The primary objective of this study was to document blood lipid profile changes following nonfocused ultrasound treatments for body contouring, elucidating clinical and biochemical effects of these devices on adipose tissue.

\section{METHODS}

\section{Device}

The MC1 device consists of ultrasound and drainage handpieces. The ultrasound handpiece delivers a dualinclined nonfocused beam of 20 to $60 \mathrm{KHz}$ on a $1 \mathrm{MHz}$ carrier wave, capable of creating a weakly focused ultrasound field within subcutaneous fat tissue at the point where the beams overlap [Figure 1].

The draining handpiece is used during both pre- and posttreatments. The former is to open the main lymph nodes to facilitate the flow of excess fluids and facilitate their removal. The latter promotes immediate lymphatic drainage of treated areas, stimulating metabolic processes that lead to natural elimination of the fat waste.

\section{Study objectives and methods}

Five males and five females (37-67 years old) in supine position were exposed to ultrasound at $20 \mathrm{KHz}$ and $3.0 \mathrm{~W} / \mathrm{cm}^{2}$ in modulated emission. Whole abdomens were treated by continuously moving an ultrasound handpiece for $30 \mathrm{~min}$, followed by a 6-min lymphatic drainage.

Waist circumferences at the level of the umbilicus and body weight were measured before and immediately after treatment. Blood lipid profiles including total cholesterol, low-density lipoprotein, high-density lipoprotein (HDL), TG, nonesterified fatty acid (NEFA) and lipoprotein-a were analyzed at baseline, every $10 \mathrm{~min}$ during treatment, and $15,30,45,60,75$, and 90 min after completion of treatment.
A paired $t$-test was performed for each group to determine the presence or absence of significant differences between baseline and post-treatment for weight, body mass index (BMI), and circumference reduction. Subjects provided written informed consent prior to participation in the study.

\section{RESULTS}

Ten subjects ( 5 males, 5 females) with an average age of 45

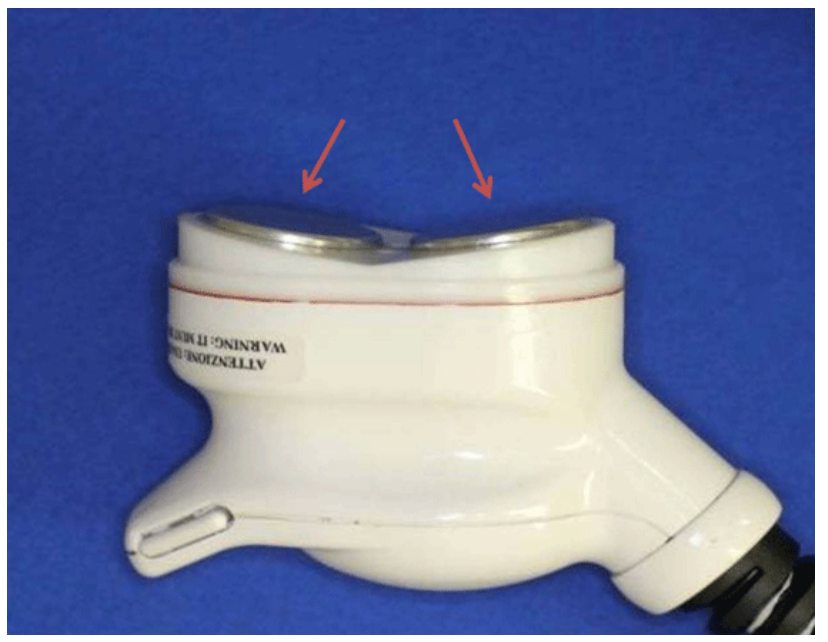

Figure 1: MC1 device. The MC1 device has an ultrasound and a zonal massage handpiece. The ultrasound handpiece with angled, nonfocused emitters deliver $1 \mathrm{MHz}$ ultrasound. In modulated emission mode, the nonfocused beams create a slightly focused ultrasound field within the tissue at the point where the beams overlap

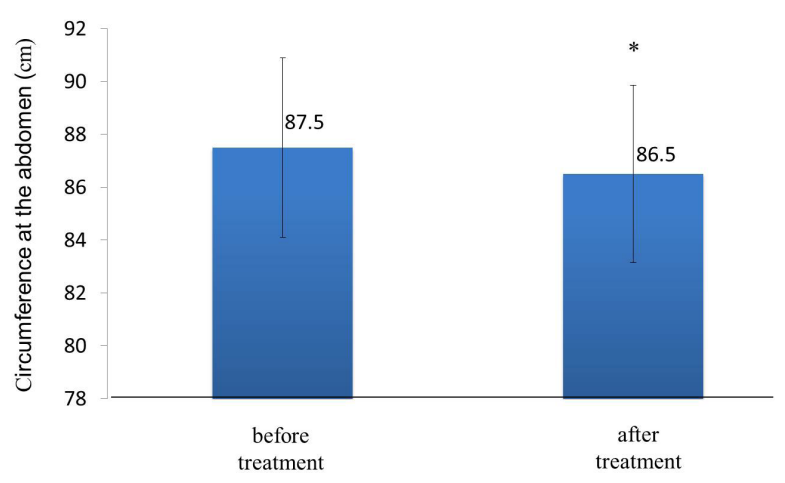

Figure 2: Summary of abdominal circumference. The circumference at the abdomen was significantly reduced ( $P<0.003)$ from baseline measurements

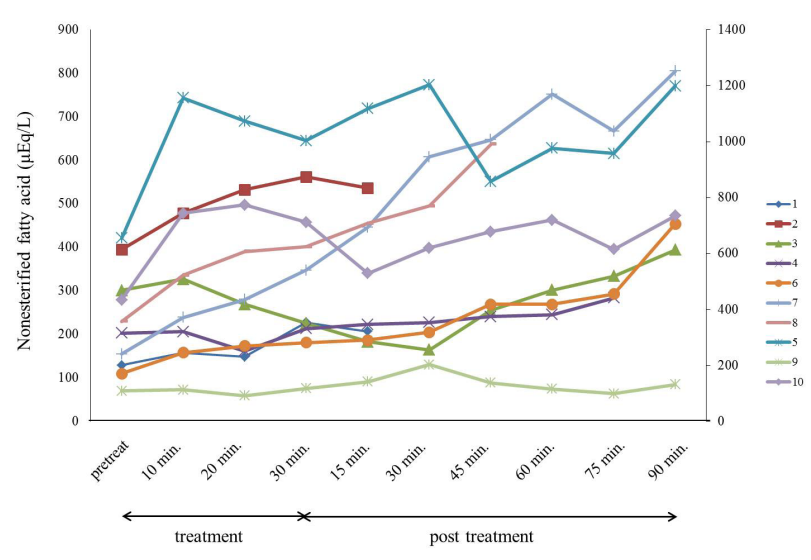

Figure 3: Blood NEFA changes in 10 subjects. Most subjects displayed increased blood NEFA levels from the early phase and had higher values at termination of the experiments. NEFA: nonesterifield fatty acid 
Table 1: Baseline data and post-treatment changes

\begin{tabular}{lcccc}
\hline & Weight $\mathbf{( k g})$ & BMI $\left(\mathbf{k g} / \mathbf{c m}^{2}\right)$ & Somatic fat rate $(\%)$ & Circumference at the abdomen $(\mathbf{c m})$ \\
\hline Before & $70.95 \pm 18.42$ & $25.82 \pm 3.49$ & $27.99 \pm 4.05$ & $88.85 \pm 10.73$ \\
After & $70.75 \pm 18.54$ & $25.75 \pm 3.51$ & $27.99 \pm 4.09$ & $88.12 \pm 10.58$ \\
\hline
\end{tabular}

The body weight, body mass index (BMI), and somatic fat rate showed slight decreases from the baseline, but they were statistically insignificant

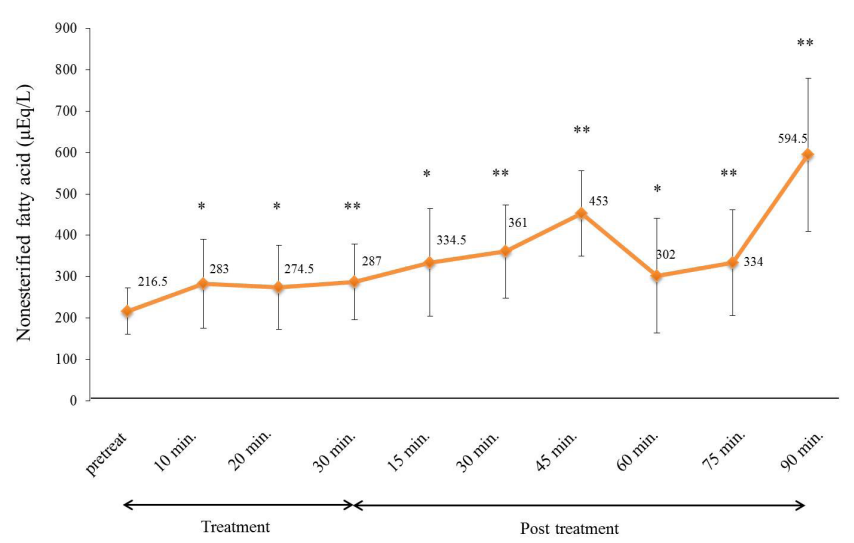

Figure 4: Summary of blood NEFA levels. Statistically significant increases from baseline NEFA were observed at any pointsduring the study period, and showed the highest values at $90 \mathrm{~min}\left({ }^{*} P<0.05\right.$, $\left.{ }^{* *} P<0.02\right)$. NEFA: nonesterifield fatty acid

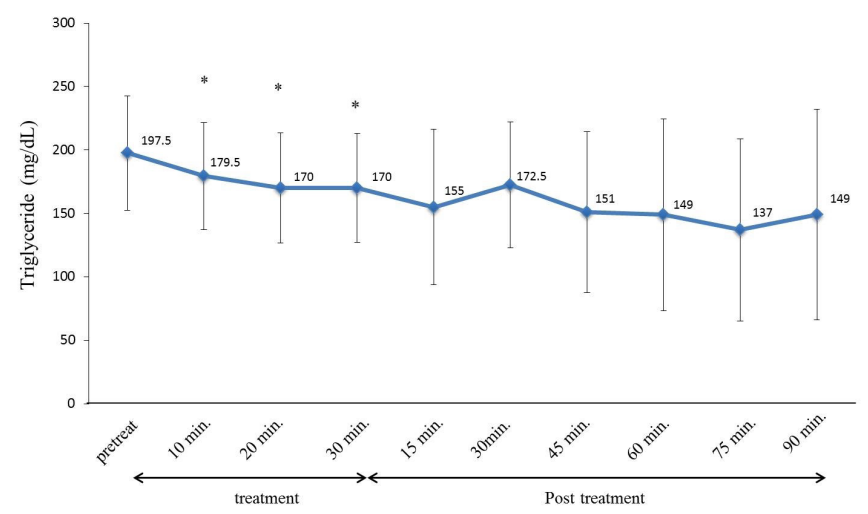

Figure 5: Summary of mean blood TG levels. TG levels up to 30 min from the beginning of the study were significantly lower, and continued to decline thereafter, albeit at a statistically insignificant level $\left({ }^{*} P<0.02\right)$. TG: triglyceride

(range 37-68 years) and an average BMI of 25.8 (range 21.3$29.8 \mathrm{~kg} / \mathrm{cm}^{2}$ ) were treated, with all subjects completing the treatments. Serial blood samples were obtained until $90 \mathrm{~min}$ after completion of the treatment from 6 subjects, until 75 min from 1 subject, until 45 min from 1 subject, and until 15 min from 2 subjects.

Circumference of the abdomen was significantly reduced $(P$ $<0.003$ ) from baseline measurements following treatment [Figure 2], whereas weight and BMI showed no statistically significant differences before and after treatment [Table 1].

While NEFA showed noticeable changes in most patients [Figure 3], other parameters did not predictably change. NEFA and TG were subsequently examined in greater detail since they appeared to have more pivotal roles in fat reduction for body contouring.

Although 2 subjects exhibited transient decreases in blood NEFA, the remaining 8 subjects demonstrated higher values at the 90 min drawing than at baseline, and concentrations were significantly higher $(P<0.05)$ throughout the periods of measurement for this cohort [Figure 4].

TG conversely showed significantly lower values $(P<0.02)$ at 10,20 , and $30 \mathrm{~min}$. A gradual decline persisted, but was not statistically significant [Figure 5].

\section{DISCUSSION}

The biological mechanisms of liporeductive effects following exposure to high-intensity focused ultrasound (HIFU) has been previously reported by many authors. HIFU, when focused within subcutaneous adipose tissue, raises the regional temperature resulting in coagulative necrosis and instantaneous cell death within the targeted area without damage to the surrounding tissue. Lipids are subsequently released from disrupted adipose tissue, and then cleared by fat metabolic pathways, and the lesion gradually heals. ${ }^{[7,8]}$ Almost all the disrupted adipocytes were resorbed within 18 weeks after treatment, resulting in an overall reduction in local fat volume without evidence of significant increases in plasma lipids..$^{[9]}$ Jewell et $a .^{[10]}$ reported that clinical laboratory tests did not reveal any abnormalities with regard to lipid profiles obtained before HIFU treatment, $1 \mathrm{~h}$ after treatment, and at weeks $1,4,8$, and 12 .

Brown et al. ${ }^{[11]}$ exploited another novel technology platform utilizing nonthermal focused ultrasound. The energy from the device was delivered as cavitation followed by mechanical destruction of cells. The term cavitation refers to a range of complex phenomena that involve the creation, oscillation, growth, and collapse of bubbles within a medium to subsequently produce mechanical energy. Little is known about the mechanism of action of nonfocused external ultrasound reducing adipose tissue.

Garcia and Schafer ${ }^{[5]}$ treated pigs with the $\mathrm{MCI}$ instrument and concluded that adipose tissue was reduced by ultrasound cavitation inducing focal alterations of the plasma membrane, and lipid leakage into interstitial space and lymphatic vessels without cell necrosis. The blood lipid profiles obtained prior to the treatments and approximately 25 to 30 min post-treatment did not show statistically significant changes in serum cholesterol, TG, or HDL.

Bani et al. ${ }^{[6]}$ concluded that lipid discharge from adipocytes was not accompanied by morphological signs of adipocyte death and disruption, or interstitial inflammation in both ex vivo and in vivo experiments; moreover, ultrasound-induced cavitation caused selective adipose cell reduction without injury to skin, vessels, nerves, or connective tissue.

Although both authors noted TG changes following ultrasonic cavitation, there was no reference to NEFA levelsin blood samples. Plasma NEFA levels significantly increased 
from baseline at all posttreatment times until 90 min after completion of the treatments.

Elevation of blood NEFA levels suggests that nonfocused ultrasound metabolized TG within fat cells to glycerol and NEFA, followed by release of NEFA into the blood stream through the lymphatic system While lymphatic drainage manipulation might shift NEFA in the lymphatic system to adipose tissue via blood circulation, blood NEFA levels increased before application of lymphatic drainage procedures. Given the half-life of NEFA of 1.7-3.0 min, such shifts of NEFA from lymphatics to the blood circulation is unlikely to maintain the high levels of NEFA until $90 \mathrm{~min}$ after drainage. It might be possible for the TG in the blood vessels within the adipose tissue being a source; however, blood vessels in adipose tissues are sparse. It is more likely that the NEFA originated from the abundant TG in the adipocytes within the adipose tissue.

There is no definitive evidence that TG can be released into inter-cellular spaces due to increased permeability of adipocyte membranes, and then degraded to NEFA and glycerol-3-phosphate in transit to the blood stream. An alternative involves lipolysis activation within adipocytes resulting in elevation of blood NEFA. TG may be metabolized within adipocytes by ultrasonic stimulations, mimicking a photochemical mechanism. The body contouring mechanism of low-level laser treatment may involve activation of cyclic adenosine monophosphate in mitochondria within adipocytes, and subsequent activation of hormone-sensitive lipases resulting in metabolism of TG in adipocytes. ${ }^{[12,13]}$

The current study suggests nonfocused ultrasound metabolizes TGs immediately after treatment, but it is unclear whether this involves intra- or extra-cellular spaces of adipocytes. Since there was no control group in the current study, but given the short half-life of NEFA, the significant elevation of blood NEFA must be due to the effects of activated lipolysis within the adipose tissue induced by ultrasound-induced cavitation.

Given the paucity of clinical or biochemical evidence for mechanisms of nonfocused ultrasound reduction of adipose tissue, further study in this field is warranted.

\section{Financial support and sponsorship}

Nil.

\section{Conflicts of interest}

There are no conflicts of interest.

\section{REFERENCES}

I. Mulholland RS, Paul MD, Chalfoun C. Noninvasive body contouring with radiofrequency, ultrasound, cryolipolysis, and low-level laser therapy. Clin Plastc Surg 201 I;38:503-20.

2. Honda T, Suzuki T, Tanaka I, Goto H, Kure K, Hishiyama J, Isago T. Clinical analysis of noninvasive body contouring by nonfocused ultrasound device. J JSAPS 2014;3:10-20. (in Japanese)

3. Teitelbaum SA, Burns JL, Kubota J, Matsuda H, Otto MJ, Shirakabe Y, Suzuki Y, Brown SA. Noninvasive body contouring by focused ultrasound: safety and efficacy of the Contour I device in a multicenter, controlled, clinical study. Plast Reconstr Surg 2007; 120:779-89.

4. Jewell ML, Solish NJ, Desilets CS. Noninvasive body sculpting technologies with an emphasis on high-intensity focused ultrasound. Aesth Plast Surg 2011;35:901-12.

5. Garcia O, Schafer M. The effects of nonfocused external ultrasound on tissue temperature and adipocyte morphology. Aesthetic Surg J 20 I 3;33: I I 7 27.

6. Bani D, Quattrini A, Freschi G, Russo GL. Histological and ultrastructural effects of ultrasound-induced cavitation on human skin adipose tissue. Plast Reconstr Surg Glob Open 2013; I:e41.

7. Haar GT, Coussios C. High intensity focused ultrasound: physical principle and devices. Int J Hyperthermia 2007;23:89-104.

8. Gadsden E, Aguilar MT, Smoller BR, Jewell ML. Evaluation of a novel highintensity focused ultrasound device for ablating subcutaneous adipose tissue for noninvasive body contouring: safety studies in human volunteers. Aesthetic Surg J 201 I;31:40 I - I0.

9. Fatemi A. High-intensity focused ultrasound effectively reduced adipose tissue. Semin Cutan Med Surg 2009;28:257-62.

10. Jewell ML, Baxter RA, Cox SE, Donofrio LM, Dover JS, Glogau RG, Kane MA, Weiss RA, Martin P, Schlessinger J. Randmized sham-controlled trial to evaluate the safety and effectiveness of a high-intensity focused ultrasound device for noninvasive body sculpting. Plast Reconstr Surg 20 I I; I 28:253-62.

II. Brown SA, Greenbaum L, Shtukmaster S, Zadok Y, Ben-Ezra S, Kushkuley L. Characterization of nonthermal focused ultrasound for noninvasive selective fat cell disruption (lysis): technical and preclinical assessment. Plast Reconstr Surg 2009; I24:92-I0I.

12. Avci P, Nyame TT, Gupta GK, Sadasivam M, Hamblin MR. Low-level laser therapy for fat layer reduction: a comprehensive review. Lasers Surg Med 2013;45:349-57.

13. McRae E, Boris J. Independent evaluation of low-level laser therapy at 635 $\mathrm{nm}$ for non-invasive body contouring of the waist, hips, thighs. Lasers Surg Med 2013;45: I-7. 